\title{
BMJ Open Cast immobilisation in situ versus open reduction and internal fixation of displaced medial epicondyle fractures in children between 7 and 16 years old. A study protocol for a randomised controlled trial
}

\author{
Tero Hämäläinen (1) , ${ }^{1}$ Matti Ahonen, ${ }^{2}$ Ilkka Helenius, ${ }^{1}$ Jenni Jalkanen, ${ }^{3}$ \\ Markus Lastikka, ${ }^{4}$ Yrjänä Nietosvaara, ${ }^{3}$ Anne Salonen, ${ }^{5}$ Juha-Jaakko Sinikumpu, ${ }^{6}$ \\ Petra Grahn (10 ${ }^{2}$
}

To cite: Hämäläinen $T$, Ahonen M, Helenius I, et al. Cast immobilisation in situ versus open reduction and internal fixation of displaced medial epicondyle fractures in children between 7 and 16 years old. A study protocol for a randomised controlled trial. BMJ Open 2021;11:e044627. doi:10.1136/ bmjopen-2020-044627

- Prepublication history for this paper is available online. To view these files, please visit the journal online (http://dx.doi org/10.1136/bmjopen-2020044627).

Received 08 September 2020 Revised 03 March 2021 Accepted 22 March 2021
Check for updates

(C) Author(s) (or their employer(s)) 2021. Re-use permitted under CC BY-NC. No commercial re-use. See rights and permissions. Published by BMJ.

For numbered affiliations see end of article.

Correspondence to Dr Tero Hämäläinen; tero.hamalainen@hus.fi

\section{ABSTRACT}

Introduction Medial epicondyle fracture of the humerus is a common injury in childhood. There is uniform agreement that minimally displaced fractures (dislocation $\leq 2 \mathrm{~mm}$ ) can be treated nonoperatively with immobilisation. Open fractures, fractures with joint incarceration or ulnar nerve dysfunction require surgery. There is no common consensus in treatment of closed medial epicondyle fractures with $>2 \mathrm{~mm}$ dislocation without joint incarceration or ulnar nerve dysfunction. We hypothesise that there is no difference in treatment outcomes between nonoperative and operative treatment.

Methods and analysis This is a multicentre, controlled, prospective, randomised noninferiority study comparing operative treatment to non-operative treatment of $>2 \mathrm{~mm}$ dislocated paediatric medial epicondyle fractures without joint incarceration or ulnar nerve dysfunction. A total of 120 patients will be randomised in 1:1 ratio to either operative or nonoperative treatment. The study will have a parallel nonrandomised patient preference arm. Operative treatment will be open reduction and internal fixation. Nonoperative treatment will be upper limb immobilisation in long arm cast for 4 weeks. Data will be collected at baseline and at each follow-up up to 2 years. QuickDASH is used as primary outcome measure. Secondary outcomes are patient-reported pain, differences in range of motion, Pediatric Quality of Life Inventory, cosmetic visual analogue scale and Mayo Elbow Performance Score.

Ethics and dissemination Ethical approval has been obtained from Helsinki University Hospital (HUS) ethical board HUS/1443/2019. Each study centre has obtained their own permission for the study. A written authorisation from legal guardian will be acquired and the child will be informed about the trial. Results of the trial will be disseminated as published articles in peer-reviewed journals.

Trial registration The trial has been registered at clinicaltrials.gov with registration number NCT04531085.
Strengths and limitations of this study

- This study is the first randomised controlled trial to examine the treatment and outcome of dislocated medial epicondyle fractures.

- This is a multicentre study with all five university hospitals in Finland participating.

- The trial has a blinded outcome assessor independent of treating and recruiting surgeons.

- Primary outcome measure (Quick DASH) is not validated for use in children.

- Treating surgeon is not blinded to the allocated treatment.

\section{INTRODUCTION}

The incidence of medial epicondyle fractures of the humerus in children and adolescents is $\geq 3: 100000$ and account for approximately $12 \%-20 \%$ of all paediatric elbow fractures, ${ }^{12} 30 \%-50 \%$ of these fractures are associated with elbow dislocation and 5\%-18\% are incarcerated. ${ }^{3}{ }^{4}$ Dysfunction of ulnar nerve has been reported in $10 \%-16 \%$ of cases. $^{3}$

Minimally displaced $(\leq 2 \mathrm{~mm})$ fractures without incarceration or ulnar nerve dysfunction are treated nonoperatively. ${ }^{5-10}$ There is no common consensus between paediatric surgeons how to treat medial epicondyle fractures with $>2 \mathrm{~mm}$ dislocation. ${ }^{11}{ }^{12}$ It has been suggested that incarcerated fractures and fractures with elbow dislocation should be treated operatively, and that competitive athletes should be treated operatively with lower threshold than children and adolescents without sporting activities. ${ }^{13}$ 
Grahn et $a l^{1}$ conducted a controlled treatment trial based on prospectively collected data from $\leq 16$ years old patients with more than $2 \mathrm{~mm}$ displaced nonincarcerated medial epicondyle fractures with a minimum follow-up of 1 year. Partial avulsion fractures were excluded, 41 were treated nonoperatively and 40 operatively. They found that neither the degree of primary fracture displacement with or without concomitant elbow dislocation nor the choice of treatment (open reduction and internal fixation (ORIF) or long arm cast) affected outcome. Normal elbow function was restored in 74/81 patients. All but one primarily nonoperatively treated patients had returned to the same or higher level of sport as preinjury, whereas six surgically treated patients had downgraded their sporting activities. Pain at medial humeral epicondyle either with direct contact or under load was reported by four nonoperatively and by six operatively treated children with normal sensation and elbow stability. ${ }^{1}$ In a systematic review of the literature regarding treatment of medial epicondyle fractures, Kamath et $a l^{14}$ found operative treatment to be superior to conservative in terms of bony union. However, the review did not show differences in terms of pain or patient-reported outcome measures between the two treatment modalities. According to the study of Lawrence $e t a l{ }^{15}$ there was no difference in outcome assessed by QuickDASH and elbow range of motion at 2 years from injury in 6 nonoperatively and 14 operatively treated athletes. Axibal $e a^{6}{ }^{6}$ showed similar results with no difference in the objective outcome in less than 1-year follow-up between 22 operated patients matched to 22 nonoperated patients.

In light of current findings, we applied for ethical review board approval to conduct a randomised noninferiority trial with the hypothesis being that conservatively treated medial epicondyle fractures fare as well as operatively treated.

\section{METHODS AND ANALYSIS \\ Study design}

The study is designed as a multicentre parallel-group noninferiority randomised controlled trial (RCT) that complies with the Consolidated Standards of Reporting Trials guidelines (figure 1). A patient preference arm will be available. Patient recruitment will be done at all university hospital areas of Finland (Helsinki, Kuopio, Oulu, Tampere, Turku). The study is coordinated by Helsinki University Central Hospital, Children's Hospital Pediatric Orthopedic Unit (HUS New Chlidren's Hospital, Stenbäckinkatu 9 C, 00029 HUS, Finland). Trial data analysts and person performing the recruitment will be unaware of the assigned treatment. The study is overseen by an external study monitor according to trial data monitoring protocol provided by $\mathrm{HUCH}$ Clinical Research Institute (Clinical Research Institute HUCH P.O Box 700, FI-00029 HUS, Helsinki, Finland, https://hyksinstituutti.fi/services/monitoring-services/?lang=en). The trial

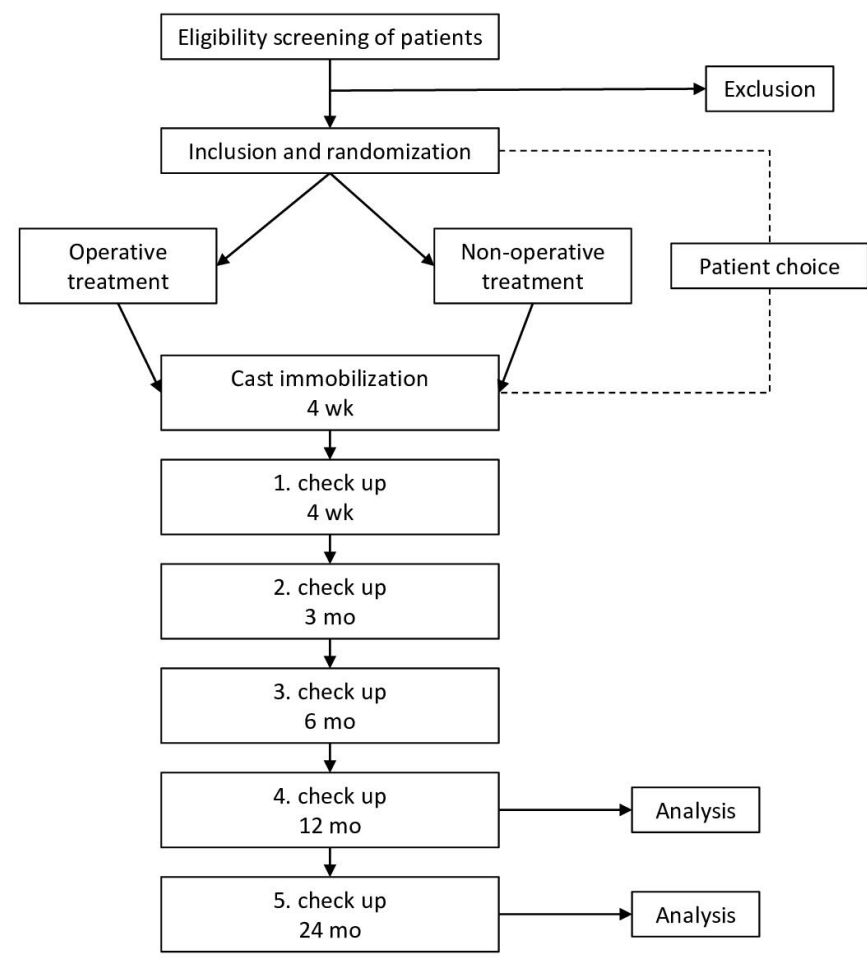

Figure 1 Flowchart of the study.

is registered at clinicaltrials.gov. Any changes in study protocol will be uploaded to the trial registry.

\section{Patient recruitment}

All patients with a medial epicondyle fracture referred to the aforementioned hospitals will be screened for eligibility by a specialist of either hand surgery, paediatric surgery, paediatric orthopaedics or orthopaedics. If inclusion criteria are met, written consent is asked from the guardian. Patients and parents are given a written informed consent regarding the trial. The patient version is age adjusted for easier understanding according to the Finnish Investigators Network for Pediatric Medicines ( www.finpedmed.fi).

\section{Inclusion criteria}

Patients aged 7-16 years presenting with a $\geq 2 \mathrm{~mm}$ displaced nonincarcerated medial epicondyle fracture with or without concomitant elbow dislocation and normal ulnar nerve function.

\section{Exclusion criteria}

Pathological fracture, open fracture, systemic bone disease, concomitant fracture or injury of the same upper limb requiring operative intervention, and other diseasepreventing participation in full follow-up regime and range of motion exercises.

\section{Randomisation}

After agreeing to participation in the trial, patients are randomised according to a computer-generated randomisation list ${ }^{17}$ to either operative or nonoperative treatment. Randomisation ratio is $1: 1$, block size is 10 . Prior to 
the recruitment, assigned arm of the RCT trial has been placed in sealed envelops. Each study centre receives a set of 10 consecutive envelopes at a time. Allocation sequence is kept at the main study centre (HUS, New Children's Hospital) where it is unavailable to recruiting physicians. Patient allocation in the trial is determined as the patient opens the assigned envelope.

\section{Patient's choice arm}

Patients who meet inclusion criteria,but refuse participation in the randomised trial are offered to choose treatment method (operative or nonoperative) and continue in a prospective parallel patient preference arm that otherwise follow the same treatment and follow-up (FU) protocol as the RCT.

\section{Baseline}

Standard anterior-posterior and lateral radiographs of the elbow will be obtained after closed reduction of the possible elbow dislocation. All participants in either the RCT or patient's choice arm undergo cone-beam or normal CT before treatment initiation. Initial fracture dislocation will be calculated from the CT scans in three planes (anterior-posterior, cranial-caudal and mediallateral) and both radiographs (anterior-posterior and lateral) using the method described by Edmonds et al. ${ }^{18}$ Date of injury, method of injury, patient's age at time of injury, sex, injured side, dominant hand and main sport or musical instrument as well as level will be documented. Motor and sensory function as well as range of motion of both upper limbs will be assessed. Carrying angle (degrees) and valgus stress test will be assessed if possible for both limbs.

\section{Intervention}

Nonoperative treatment means upper limb immobilisation with forearm in neutral prosupination with a long arm cast for 4 weeks. Treatment is started after baseline examination.

Operative treatment is scheduled after baseline examination and is to be done within 7 days from injury. During sedation, both elbows are stress-tested using the valgus stress test, any instability is documented, carrying angle of both elbows are measured. Procedure of preference is ORIF with cannulated nonresolvable $4.0 \mathrm{~mm}$ screw with or without washer. If the fracture fragment is too small or fragmented for screw fixation, $1.6 \mathrm{~mm}-1.8 \mathrm{~mm}$ Kirshner-wires and/or bone anchor are used. After fracture fixation, the injured side is again stress tested. Radiographs (anterior-posterior and lateral) documenting the fixation are taken. Long arm cast with forearm in neutral prosupination is applied for 4 weeks. Time from injury to surgery, fixation method, length of surgery (min) and surgeon's level of training (consultant, registrar) will be documented. Hardware is not routinely removed.

All patients will receive a written exercise plan explaining the active and passive range of motion (ROM) exercises that are to be performed at a minimum three times per day from cast removal. Physiotherapy will be offered if guardians and/or patients feel that no progress in ROM after 2 weeks of home exercises.

\section{Blinding}

This trial tests a clinical intervention that is not suitable for protection against treatment bias. Recruiter will be blinded. Consultant on duty will perform randomisation and allocation. Nonoperative treatment will be started immediately after recruitment. ORIF will be performed by surgeon on duty. Surgeon is not blinded. Trial data are collected at each appointment at the outpatient clinic by a physician not related to the trial. Statistician analysing trial data are blinded to treatment group.

\section{Outcome measure}

Follow-up is set at 1, 3, 6, 12 and 24 months from initiation of treatment with the option of ending the FU at 12 months if patients are pain free with full ROM in relation to uninjured side. Elbow standard radiographs (anteriorposterior and lateral) are taken at each appointment from 3 months on until bone union is achieved or trial ends (table 1).

Patients will be examined at the paediatric orthopaedic outpatient clinic. On each appointment, active and passive ROM of both upper limbs (elbow extensionflexion, prosupination, wrist extension-flexion) as well as carrying angle are measured using a goniometer. Stability of both elbows is assessed using the moving valgus test $\mathrm{t}^{19}$ and the valgus stress test. ${ }^{20}$ Distal sensation is examined by Semmes-Weinstein monofilaments. ${ }^{21}$ Signs of cold intolerance will be assessed. Grip strength is measured with a dynamometer.

Patients and guardians are requested to answer the following patient-reported outcome measures at each appointment; QuickDASH, ${ }^{22}$ Pediatric Quality of Life Inventory (PedsQL), PedsQL Pediatric Pain Questionnaire, ${ }^{23}$ cosmetic visual analogue scale (VAS 0-100) and Mayo Elbow Performance Score (MEPS). ${ }^{24}$

Time of returning to main sport or music and its level will be documented (weeks). Any adverse effects (wound infection, nerve damage) are documented as well as hardware problems and possible hardware removal as well as conversion of treatment during FU (cast to ORIF or ligament reconstruction).

Allthough QuickDash is not validated for use in under 18-year olds, it was selected as it has been used in the literature in evaluating results of fracture healing in adolescents, and it is available in both national languages (Finnish and Swedish). ${ }^{25} 26$

\section{Primary outcome}

Statistically significant difference in QuickDASH score is $6.8^{2526}$ at 12 months FU.

\section{Secondary outcome}

Difference in active ROM in comparison to uninjured arm, PedsQL, PEDS QL Pain module, Cosmetic VAS, MEPS, need for additional procedures. 


\begin{tabular}{|c|c|c|c|c|c|c|c|}
\hline & Baseline & $\begin{array}{l}\text { Treament } \\
\text { Day } 0\end{array}$ & $\begin{array}{l}\text { 1.Check-up } \\
4 \text { weeks }\end{array}$ & $\begin{array}{l}\text { 2.Check-up } \\
3 \text { months }\end{array}$ & $\begin{array}{l}\text { 3.Check-up } \\
6 \text { months }\end{array}$ & $\begin{array}{l}\text { 4.Check-up } \\
12 \text { months }\end{array}$ & $\begin{array}{l}\text { 5.Check-up } \\
24 \text { months }\end{array}$ \\
\hline $\begin{array}{l}\text { Diagnosis, } \\
\text { eligibility }\end{array}$ & $\mathbf{x}$ & & & & & & \\
\hline Randomisation & $\mathbf{x}$ & & & & & & \\
\hline $\begin{array}{l}\text { Surgery or } \\
\text { non-operative } \\
\text { treatment }\end{array}$ & & $\mathbf{x}$ & & & & & \\
\hline $\begin{array}{l}\text { Physical } \\
\text { examination }\end{array}$ & $\mathbf{x}$ & & $\mathbf{x}$ & $\mathbf{x}$ & $\mathbf{x}$ & $\mathbf{x}$ & $\mathbf{x}$ \\
\hline Questionnaires & & & $x$ & $x$ & $\mathbf{x}$ & $x$ & $x$ \\
\hline $\begin{array}{l}\text { Computer } \\
\text { tomography }\end{array}$ & $\mathbf{x}$ & & & & & & \\
\hline $\begin{array}{l}\text { Standard } \\
\text { radiograph }\end{array}$ & $x$ & & $\mathbf{x}$ & $x$ & $x$ & $x$ & $x$ \\
\hline
\end{tabular}

\section{Sample size}

Based on the results of Kazmers et $a l^{25}$ and Aasheim and Finsen, ${ }^{26}$ we assume clinically significant difference between the groups to be 6.8 and the SD of the QuickDASH score to be 10 points. With 0.05 significance level and $80 \%$ power, a noninferiority comparison would require 27 patients per group. Allowing a 20\% dropout rate, the required sample would be 30 patients per group.

For subgroup analysis (less than 12 years vs 12 years and over), 30 patients per age group need to be collected. Assuming 50-50 split in the patients between the age groups, the sample size would be 60 per ORIF and nonoperated equalling a total of 120 patients.

\section{Statistical analysis}

Data will be analysed by using the Wilcoxon rank-sum test in Python V.3.8. (Python Software Foundation, Wilmington, Delaware, USA). Our null hypothesis is that there is no difference in outcome between nonoperative versus ORIF. Level of significance is set at $\mathrm{p}<0.05$.

Both treatment groups will be internally analysed for differences in primary outcome regarding age (less than 12 years vs 12 years and over) at time of injury and amount of initial fracture displacement $(\mathrm{mm})$. Depending on group size, patient choice arm can be merged for analysis to same RCT group.

\section{Patient and public involvement}

Patients, caregivers or public were neither involved in the development of the research questions nor the planning of the study design. They are neither involved in the recruitment nor conduct of the study. Results of the study are published only in peer-reviewed journals, no other information of the results of the study are provided to the patients or caregivers. Patients or caregivers will not take part in assessment regarding possible burden of the interventions of this study.

\section{ETHICS AND DISSEMINATION}

There is no common consensus for dislocated $(>2 \mathrm{~mm})$ medial epicondyle fractures. Treatment method varies by clinic and treating surgeon. Both ORIF and long arm cast are well-established treatment methods for humeral medial epicondyle fractures. If at any point an imminent problem in healing is observed, warranting a change in the treatment regimen, this will be done at the discretion of the treating physician regardless of the initial treatment allocation. The participants will be treated according to our best knowledge during and after the trial. Patients will not receive any compensation for participation. The Finnish Patient Insurance Centre will provide compensation for treatment injuries.

We have obtained national ethical approval from Helsinki University Hospital (HUS) ethical board HUS/1443/2019. A local permission to conduct the trial will be obtained by each study centre (Kuopio University Hospital, Oulu University Hospital, Tampere University Hospital and Turku University Hospital). A written authorisation from guardian will be acquired and child will be informed about the trial. Results of the trial will be disseminated as published articles in peerreviewed journals. Authorship will follow the International Committee of Medical Journal Editors (ICMJE) recommendations. ${ }^{27}$

\section{Time schedule}

Last patient FU is expected by the end of 2023 and publication by the end of 2024 .

\section{CONCLUSION}

The goal of this study is to compare two well-established treatment methods of dislocated nonincarcerated humeral medial epicondyle fractures in 7-16-year-old patients. 


\section{Author affiliations}

${ }^{1}$ Department of Orthopedics and Traumatology, University of Helsinki and Helsinki University Hospital, Helsinki, Uusimaa, Finland

${ }^{2}$ Department of Pediatric Orthopedics and Traumatology, University of Helsinki and Helsinki University Hospital, Helsinki, Uusimaa, Finland

${ }^{3}$ Department of Pediatric Surgery, Kuopio University Hospital, Kuopio, Pohjois-Savo, Finland

${ }^{4}$ Department of Paediatric Orthopaedic Surgery, TYKS Turku University Hospital, Turku, Varsinais-Suomi, Finland

${ }^{5}$ Department of Pediatric Surgery, Tampere University Hospital, Tampere, Finland

${ }^{6}$ Department of Children and Adolescents, Oulu University Hospital, Oulu, Finland

Contributors TH, MA and PG have conceived and designed the study, performed the analysis and written the paper. $\mathrm{IH}, \mathrm{JJ}, \mathrm{ML}, \mathrm{YN}, \mathrm{AS}$ and J-JS have participated in writing the paper.

Funding The authors have not declared a specific grant for this research from any funding agency in the public, commercial or not-for-profit sectors.

Competing interests $\mathrm{IH}$ reports grants from Medtronic and Stryker. $\mathrm{IH}$ is consulting surgeon at Medtronic. J-JS is consulting surgeon at Bioretec Ltd. None of the other authors reports any conflict of interest.

Patient and public involvement Patients and/or the public were not involved in the design, or conduct, or reporting, or dissemination plans of this research.

Patient consent for publication Not required.

Provenance and peer review Not commissioned; externally peer reviewed.

Open access This is an open access article distributed in accordance with the Creative Commons Attribution Non Commercial (CC BY-NC 4.0) license, which permits others to distribute, remix, adapt, build upon this work non-commercially, and license their derivative works on different terms, provided the original work is properly cited, appropriate credit is given, any changes made indicated, and the use is non-commercial. See: http://creativecommons.org/licenses/by-nc/4.0/.

\section{ORCID iDs}

Tero Hämäläinen http://orcid.org/0000-0003-2807-0230

Petra Grahn http://orcid.org/0000-0003-3018-8270

\section{REFERENCES}

1 Grahn P, Hämäläinen T, Nietosvaara Y, et al. Comparison of outcome between nonoperative and operative treatment of medial epicondyle fractures. Acta Orthop 2021;92:114-9.

2 Flynn J, Skaggs D, Waters P. Rockwood \& Wilkins Fractures in Children. 8th edn. Wolters Kluwer, 2014.

3 Louahem DM, Bourelle S, Buscayret F, et al. Displaced medial epicondyle fractures of the humerus: surgical treatment and results. A report of 139 cases. Arch Orthop Trauma Surg 2010;130:649-55.

4 Hyvönen $\mathrm{H}$, Korhonen L, Hannonen J, et al. Recent trends in children's elbow dislocation with or without a concomitant fracture. BMC Musculoskelet Disord 2019;20:294.

5 Gottschalk HP, Eisner E, Hosalkar HS. Medial epicondyle fractures in the pediatric population. J Am Acad Orthop Surg 2012;20:223-32.

6 Smith FM. Medial epicondyle injuries. J Am Med Assoc 1950;142:396-402.
7 Blount W. Fractures in children. London: Williams \& Wilkins, 1955.

8 Maylahn DJ, Fahey JJ. Fractures of the elbow in children; review of three hundred consecutive cases. J Am Med Assoc 1958; 166:220-8.

9 Bede WB, Lefebvre AR, Rosman MA. Fractures of the medial humeral epicondyle in children. Can J Surg 1975;18:137-42.

10 Tarallo L, Mugnai R, Fiacchi F, et al. Pediatric medial epicondyle fractures with intra-articular elbow incarceration. $J$ Orthop Traumatol 2015;16:117-23.

11 Lee $\mathrm{H}-\mathrm{H}$, Shen $\mathrm{H}-\mathrm{C}$, Chang J-H, et al. Operative treatment of displaced medial epicondyle fractures in children and adolescents. $J$ Shoulder Elbow Surg 2005;14:178-85.

12 Pezzutti D, Lin JS, Singh S, et al. Pediatric medial epicondyle fracture management: a systematic review. J Pediatr Orthop 2020;40:e697-702.

13 Baety J, Kasser J. Chapter 15 in Rockwood \& Wilkins Fractures in Children. 8th edn. Philadelphia, PA: Lippicott Williams \& Wilkins, 2014.

14 Kamath AF, Baldwin K, Horneff J, et al. Operative versus nonoperative management of pediatric medial epicondyle fractures: a systematic review. J Child Orthop 2009;3:345-57.

15 Lawrence JTR, Patel NM, Macknin J, et al. Return to competitive sports after medial epicondyle fractures in adolescent athletes: results of operative and nonoperative treatment. Am J Sports Med 2013;41:1152-7.

16 Axibal DP, Carry P, Skelton A, et al. No difference in return to sport and other outcomes between operative and Nonoperative treatment of medial Epicondyle fractures in pediatric upper-extremity athletes. Clin J Sport Med 2018;1.

17 Urbaniak GC, Plous S. Research Randomizer 2011.

18 Edmonds EW. How displaced are "nondisplaced" fractures of the medial humeral epicondyle in children? Results of a threedimensional computed tomography analysis. J Bone Joint Surg Am 2010;92:2785-91.

19 O'Driscoll SWM, Lawton RL, Smith AM. The "moving valgus stress test" for medial collateral ligament tears of the elbow. Am J Sports Med 2005;33:231-9.

20 Flynn T. User's 220 guide to the musculoskeletal examination: Fundamentals for the evidence-based clinician. Buckner, Kentucky: Evidence in Motion, 2008.

21 Bell-Krotoski J. "Pocket filaments" and specifications for the semmes-weinstein monofilaments. Journal of Hand Therapy 1990;3:26-31.

22 Beaton DE, Wright JG, Katz JN, et al. Development of the QuickDASH: comparison of three item-reduction approaches. J Bone Joint Surg Am 2005;87:1038-46.

23 Varni JW, Seid M, Rode CA. The PedsQL: measurement model for the pediatric quality of life inventory. Med Care 1999;37:126-39.

24 Morrey B. The elbow and its disorders. Philadelphia: WB Saunders, 1993.

25 Kazmers NH, Quu Y, Yoo M, et al. The minimal clinically important difference of the PROMIS and QuickDASH instruments in a Nonshoulder hand and upper extremity patient population. $J$ Hand Surg Am 2020;45:399-407.

26 Aasheim T, Finsen V. The DASH and the QuickDASH instruments. normative values in the general population in Norway. $J$ Hand Surg Eur Vol 2014;39:140-4.

27 International Committee of medical Journal editors. defining the role of authors and contributors. Available: http://www.icmje.org/ recommendations/browse/roles-and-responsibilities/defining-therole-of-authors-and-contributors.html 